This item was submitted to Loughborough's Research Repository by the author.

Items in Figshare are protected by copyright, with all rights reserved, unless otherwise indicated.

\title{
Orientation-field models for polycrystalline solidification: grain coarsening and complex growth forms
}

PLEASE CITE THE PUBLISHED VERSION

https://doi.org/10.1016/j.jcrysgro.2016.06.040

PUBLISHER

(C) Elsevier

VERSION

AM (Accepted Manuscript)

\section{PUBLISHER STATEMENT}

This work is made available according to the conditions of the Creative Commons Attribution-NonCommercialNoDerivatives 4.0 International (CC BY-NC-ND 4.0) licence. Full details of this licence are available at: https://creativecommons.org/licenses/by-nc-nd/4.0/

\section{LICENCE}

CC BY-NC-ND 4.0

\section{REPOSITORY RECORD}

Korbuly, Balint, Tamas Pusztai, Gyula I. Toth, Herve Henry, Mathis Plapp, and Laszlo Granasy. 2019. "Orientation-field Models for Polycrystalline Solidification: Grain Coarsening and Complex Growth Forms". figshare. https://hdl.handle.net/2134/27266. 


\title{
Orientation-field models for polycrystalline solidification: grain coarsening and complex growth forms
}

\author{
Bálint Korbuly, ${ }^{1, \S}$ Tamás Pusztai, ${ }^{1}$ Gyula I. Tóth, ${ }^{1,2}$ Hervé Henry, ${ }^{3}$ Mathis Plapp, ${ }^{3}$ and László Gránásy ${ }^{1,4}$ \\ ${ }^{1}$ Institute for Solid State Physics and Optics, Wigner Research Centre for Physics, P O Box 49, H-1525 Budapest, Hungary \\ ${ }^{2}$ Department of Physics and Technology, University of Bergen, Allégaten 55, 7000 Bergen, Norway \\ ${ }^{3}$ Physique de la Matière Condensée, École Polytechnique, CNRS 91128, Palaiseau, France \\ ${ }^{4}$ BCAST, Brunel University, Uxbridge, Middlesex, UB8 3PH, UK \\ $\S$ Corresponding author: korbuly.balint@wigner.mta.hu
}

We compare two versions of the phase-field theory for polycrystalline solidification, both relying on the concept of orientation fields: one by Kobayashi et al. [Physica D 140 (2000) 141] and the other by Henry et al. [Phys. Rev. B 86 (2012) 054117]. Setting the model parameters so that the grain boundary energies and the time scale of grain growth are comparable in the two models, we first study the grain coarsening process including the limiting grain size distribution, and compare the results to those from experiments on thin films, to the models of Hillert, and Mullins, and to predictions by multiphase-field theories. Next, following earlier work by Gránásy et al. [Phys. Rev. Lett. 88 (2002) 206105; Phys. Rev. E 72 (2005) 011605], we extend the orientation field to the liquid state, where the orientation field is made to fluctuate in time and space, and employ the model for describing of multi-dendritic solidification, and polycrystalline growth, including the formation of "dizzy" dendrites disordered via the interaction with foreign particles.

Keywords: A1: Polycrystalline Solidification, Polycrystalline Growth, Growth Front Nucleation, Orientation Field Models, Multiphase-field Models, Computational Materials Science

\section{Introduction}

A substantial fraction of the solid materials human civilization relies on is polycrystalline. This includes technical alloys, minerals, medicines, certain types of food, etc. Polycrystalline matter is composed of crystallites, whose size, shape, and composition distributions define its properties. These features are determined during solidification, and may be influenced by the conditions of freezing and subsequent heat treatments. The desire to control these features drives materials scientists towards investigating the associated phenomena.

It has been established experimentally that during the time evolution of thin metallic films, the distribution of grain sizes approaches a steady state characterized by a "limiting grain size distribution" (LGSD) [1], which can be well approximated by a lognormal distribution as proposed by Feltham [2]. Other models by Mullins [3] and Hillert [4] predict different functional forms for LGSD in 2D. The problem of grain coarsening has been addressed by multi-order parameter [5-7] and multiphase-field $[8,9]$ models that assign a separate order parameter or phase field to every crystal grain. While the predictions are fairly close to each other, they seem to differ perceptibly from the experiments. Remarkably, a simple dynamical density functional theory, known as the "Phase-Field Crystal" (PFC) model $[10,11]$, recovers the experimental LGSD nearly perfectly [12]. As in the experiments, the effect of different physical phenomena shaping the LGSD cannot be separated in the PFC simulations. In this situation, a comparison of different models may contribute to the identification of the governing phenomena. Along this line, herein, we investigate the LGSD emerging from phase-field models that rely on an orientation field, $\theta(\mathbf{r}, t)$, to describe the crystallographic orientation of the individual grains [13].

The orientation field models can be divided into two categories: the earlier approaches introduced a free energy term proportional to $|\nabla \theta|$, as proposed by Kobayashi and coworkers
[14-16] (KWC model henceforth). Its variants have been applied for a broad range of solidification phenomena including the formation of multiple dendrites [17, 18], disordered ("dizzy") dendrites [19], grain rotation [15], fractal growth forms [18], and spherulitic solidification [20, 21]. Another approach has been put forward recently by Henry et al. [22] (HMP model henceforth), in which the free energy contribution from the orientation field is proportional to $(\nabla \theta)^{2}$, with a coefficient that is singular in the solid phase. It has not yet been established whether the latter model is capable of reproducing polycrystalline growth forms that result from the emergence of new grains at the perimeter as growth proceeds. Following [20, 21], we term this phenomenon of creating new grains at the perimeter Growth Front Nucleation (GFN).

In the present paper we address two problems: (i) we investigate the form of LGSD in the orientation field models (KWC and HMP); and (ii) we explore whether it is possible to capture the GFN mechanisms leading to polycrystalline growth within the HMP model. For the latter purpose we employ a binary version of the HMP model [13].

\section{The orientation field models}

In the phase-field models relying on the concept of orientation field, the local state of matter is characterized by a coarsegrained structural order parameter, the phase field $\phi$, monitoring the crystal-liquid transition, and an additional field, $\theta$ (a scalar angular field in 2D), which shows the local crystallographic orientation relative to the laboratory frame. These fields are usually coupled to other slowly evolving fields, such as the concentration field, $c$. The free energy of the inhomogeneous system can then be expressed as

$$
F=\int d \mathbf{r}\left\{\frac{\varepsilon_{\phi}^{2} s^{2}}{2}(\nabla \phi)^{2}+W g(\phi)+f_{\text {bul }}(\phi, c)+f_{\text {ori }}(\phi, \nabla \theta)\right\}
$$


where $\varepsilon \phi^{2}$ and $W$ are parameters related to the excess free energy and the thickness of the equilibrium solid-liquid interface. The function $s=s(\vartheta, \theta)=1+s_{0} \cos [k \vartheta-2 \pi \theta]$ assigns anisotropy to the interface free energy (of $k$-fold symmetry). Here $\vartheta=\arctan \left[(\nabla \phi)_{\mathrm{y}} /(\nabla \phi)_{\mathrm{x}}\right]$ is the orientation of the interface, whereas $\theta$ is the local crystallographic orientation. Both $\vartheta$ and $\theta$ are normalized so that they vary between 0 and 1 . The bulk free energy density, $f_{\text {bulk }}$, varies between those for the solid and liquid phases ( $f_{s}$ and $f_{l}$, respectively) as prescribed by the interpolating function $p(\phi)$ :

$$
\begin{gathered}
f_{\text {bul k }}=p(\phi) f_{s}+(1-p(\phi)) f_{l} \\
p(\phi)=\phi^{3}\left(10-15 \phi+6 \phi^{2}\right)
\end{gathered}
$$

In the single component case (Sections 4.1 and 4.2), $f_{s}$ and $f_{l}$, stand for the free energy densities of the pure material at the given temperature, whereas in the binary case (Section 4.3), $f_{s}$ and $f_{l}$ were taken from the ideal solution model.

The orientation field models KWC and HMP differ in the form of the orientational free energy density, $f_{\text {ori }}$ as follows:

$$
\begin{gathered}
f_{\text {or } \mathrm{i}}^{\mathrm{KWC}}=r(\phi)\left[H_{1}|\nabla \theta|+H_{2}|\nabla \theta|^{2}\right] \\
f_{\text {ori }}^{\mathrm{HMP}}=q(\phi) H|\nabla \theta|^{2}
\end{gathered}
$$

where $r(\phi)=\phi^{4}$, and $q(\phi)=\left(7 \phi^{3}-6 \phi^{4}\right) /(1-\phi)^{3}$, while the coefficients $H$ tune the strength of the respective gradient terms.

The time evolution of the three fields are described by the usual variational equations of motion (EOMs):

$$
\begin{aligned}
& \frac{\partial \phi}{\partial t}=-M_{\phi} \frac{\delta \phi}{\delta t}+\zeta_{\phi} \\
& \frac{\partial \phi}{\partial t}=\nabla\left\lfloor M_{c} \nabla \frac{\delta F}{\delta c}\right\rfloor \\
& \frac{\partial \theta}{\partial t}=-M_{\theta} \frac{\delta \theta}{\delta t}+\zeta_{\theta}
\end{aligned}
$$

where $\zeta_{i}$-s are noise terms representing the thermal fluctuations.

The mobilities $M_{\phi}$ and $M_{\mathrm{c}}$ are defined in [13, 20, 21] and to allow for the freezing of orientational disorder in the solid, we used

$$
\begin{gathered}
M_{\theta}^{\mathrm{KWC}}=M_{\theta, 0}^{\mathrm{KWC}}(1-p(\phi)) \\
M_{\theta}^{\mathrm{HMP}}=M_{\theta, 0}^{\mathrm{HMP}}(1-p(\phi))(1-\phi)^{3},
\end{gathered}
$$

where the $M_{\theta}^{i}$-s are the mobilities of the orientation field in the liquid phase. The $(1-\phi)^{3}$ multiplier in Eq. (10) compensates for the diverging driving force of orientational ordering, which emerges from the singular coupling function $q(\phi)$ in Eq. (5). As a result, similar time scales are expected for the evolution of the orientation field in the two models.

\section{Numeric solutions and materials parameters}

The EOMs were solved numerically using finite difference discretization with explicit forward Euler time stepping on rectangular grids of various sizes, while prescribing periodic boundary conditions. Parallel codes were implemented for CPU clusters and GPU cards. The computations were performed on a CPU cluster consisting of $740 \mathrm{CPU}$ cores, and on 35 GPU cards of various types.
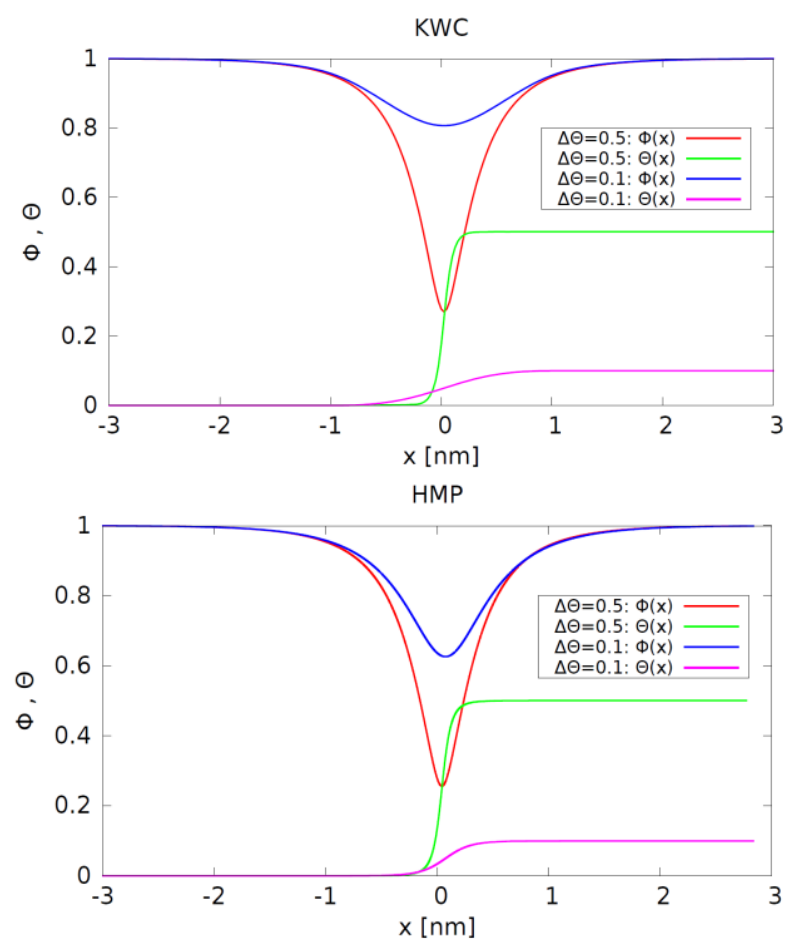

Fig. 1. Phase field and orientation field profiles through the grain boundary in the KWC and HMP models, corresponding to $\Delta \theta=0.5$ and 0.1 in Figure 2.

In computations for the single component case, properties of pure $\mathrm{Ni}$ were used, whereas in the binary case we employed the data for $\mathrm{Cu}-\mathrm{Ni}$ system taken from Ref. [17].

\section{Results and Discussion}

\subsection{Grain boundaries in the orientation field models}

First, we investigate the structure of the solid-solid interface (grain boundary) at two substantially different misorientations $(\Delta \theta=0.1$ and 0.5$)$ of the neighboring crystals. The results for the KWC and HMP models are shown in Figs. 1 and 2, respectively. In agreement with previous work [16, 22], a misfit dependent depression of the phase field is observed at the grain

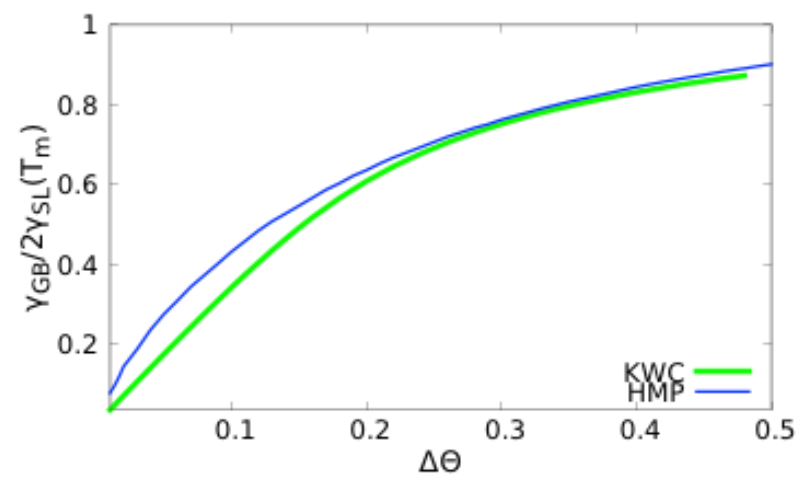

Fig. 2. Grain boundary energy in the KWC and HMP models as function of the misorientation angle $\Delta \theta$. A good match of the two curves is obtained with the choice of $H_{1}=4.6 \mathrm{~J} / \mathrm{m}^{2}$ and $H_{2}=H=9.37 \times 10^{-10}$ $\mathrm{J} / \mathrm{m}$. For high angles, the grain boundary energies approach the value of twice the solid-liquid interface energy $\gamma \mathrm{sL}$, as expected. 


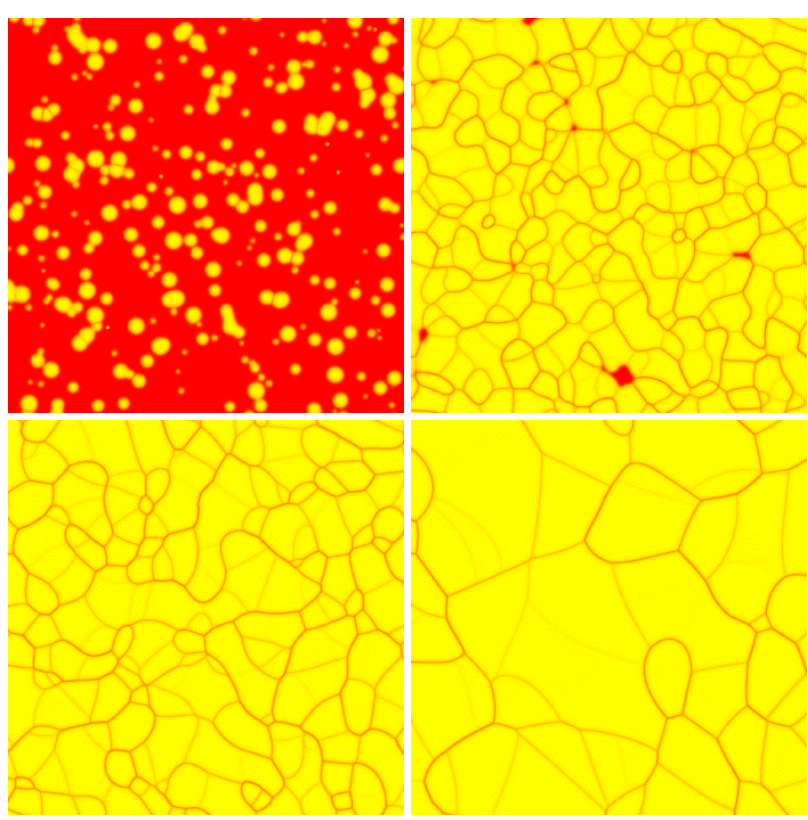

Fig. 3. Nucleation, growth, and grain coarsening in $2 \mathrm{D}$ within the KWC model. Snapshots of the phase field taken at $5 \times 10^{4}, 1.5 \times 10^{5}$, $5 \times 10^{5}$, and $4 \times 10^{6}$ time steps are shown. The properties of $\mathrm{Ni}$ have been used. $1024^{2}$ sections of a $4096^{2}$ simulation are displayed.

boundary. The two models display qualitatively similar behavior, although the HMP model has a slightly sharper interface under the same conditions. We found that the respective grain boundary energies are comparable.

\subsection{Grain coarsening}

To study grain coarsening, we produced a polycrystalline initial state via placing supercritical seeds randomly in time and space in the simulations box analogously to the procedure proposed by Simmons et al. [23], which process lead eventually to a full crystallization of the simulation box. We used the depressions in the phase-field map to identify the crystal grains (Fig. 3 ), and determined their size distribution. In agreement with previous results, after a transient we have observed a steady state limiting grain size distribution (LGSD). The results for the KWC and HMP models are compared with the experiments in Fig. 4 upper panel. While the qualitative features of the LGSD from the two model are comparable, yet they deviate from the experiment perceptibly. For comparison, we also present the theoretical predictions by Mullins [3, 12] and Hillert [4]. Remarkably, the predictions from the orientation field models are considerably closer to the experiments than these theories (Fig. 4, central panel). We also recall results from the multiphasefield theories by Kim et al. [8], Schaffnit et al. [9], and Tóth et al. [24] (Fig. 4 lower panel). Apparently, the LGSDs from the orientation field theories are comparable to those from the multiphase-field models of Kim et al. and Schaffnit et al. Of these coarse grained phase-field models, apparently the one by Tóth et al. approximates the experimental results the best, still it is much less successful than the PFC [12]: it overestimates the population of the small clusters considerably, and underestimates the height of the peak. Summarizing, further work is needed to clarify which feature of the PFC model makes it possible to recover the experimental LGSD so accurately. This goal might be achieved by varying the model parameters in the
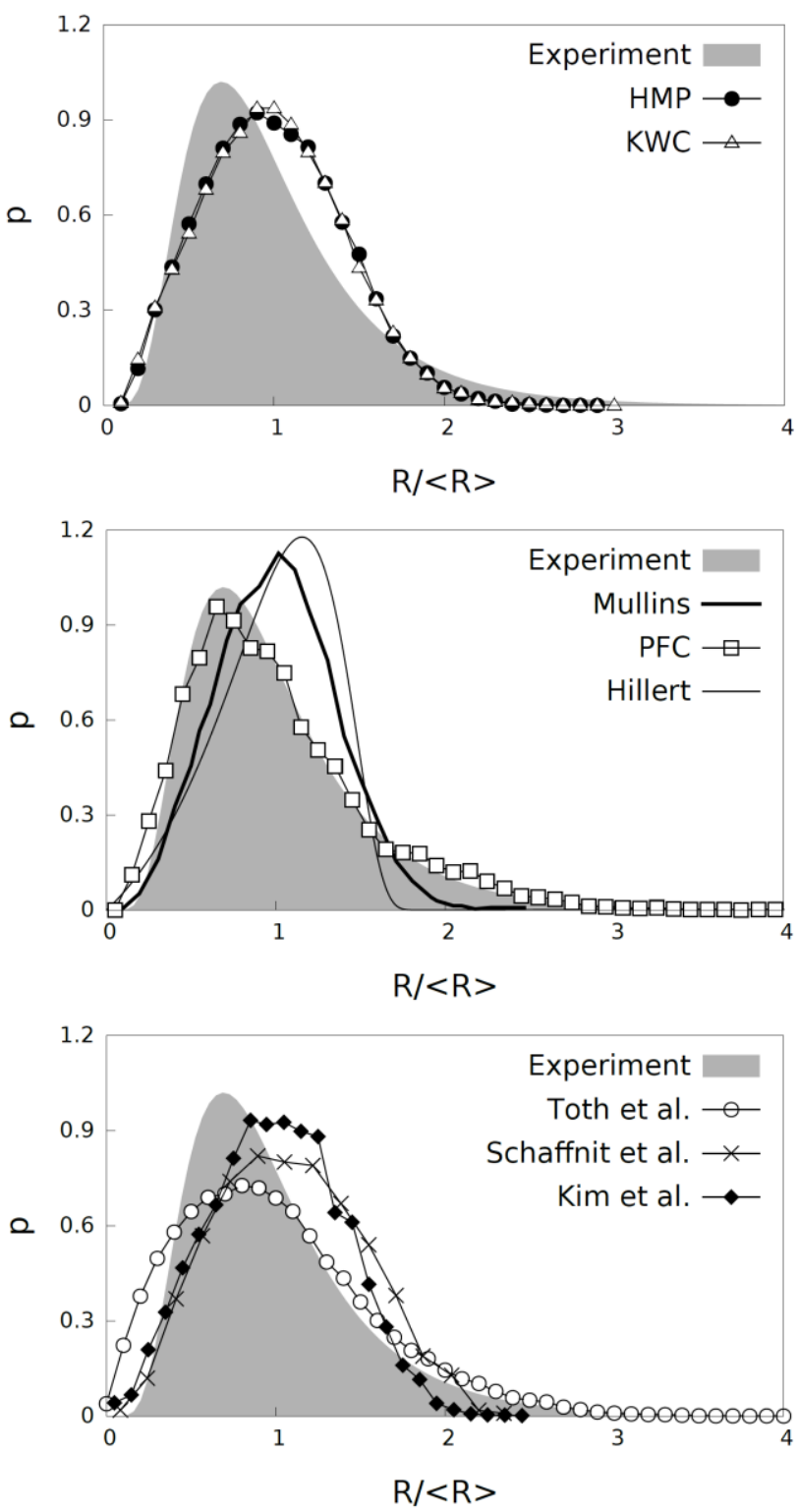

Fig. 4. Limiting grain size distribution (LGSD) for grain coarsening in 2D. Upper panel: orientation field theories (KWC and HMP) vs. experiment; central panel: theoretical predictions by the models of Mullins, Hillert, and PFC simulations [12] vs. experiment; lower panel: multiphase-field theories vs. experiment. The experiments on thin films are represented by a lognormal distribution that describes the experimental data accurately (see Fig. A1 by Barmak et al. [1], $\sigma$ $=0.5$, and $\mu=-0.12$ ).

other models, while observing the respective changes in the LGSD.

\subsection{Polycrystalline solidification in HMP}

While the KWC model and its descendants have been widely used to address various aspects of polycrystalline solidification [13-21], there are only few studies [22] regarding the abilities of the HMP model in this area. Extending the orientation field to the liquid and making it fluctuate in time and space as in Ref. [21], noise induced nucleation of crystals with a random orientation has been realized. Snapshots of the orientation field of such process is shown for $\mathrm{Cu}-\mathrm{Ni}$ in Fig. 5. [An anisotropic interface free energy of four-fold symmetry $(k=4)$ has 


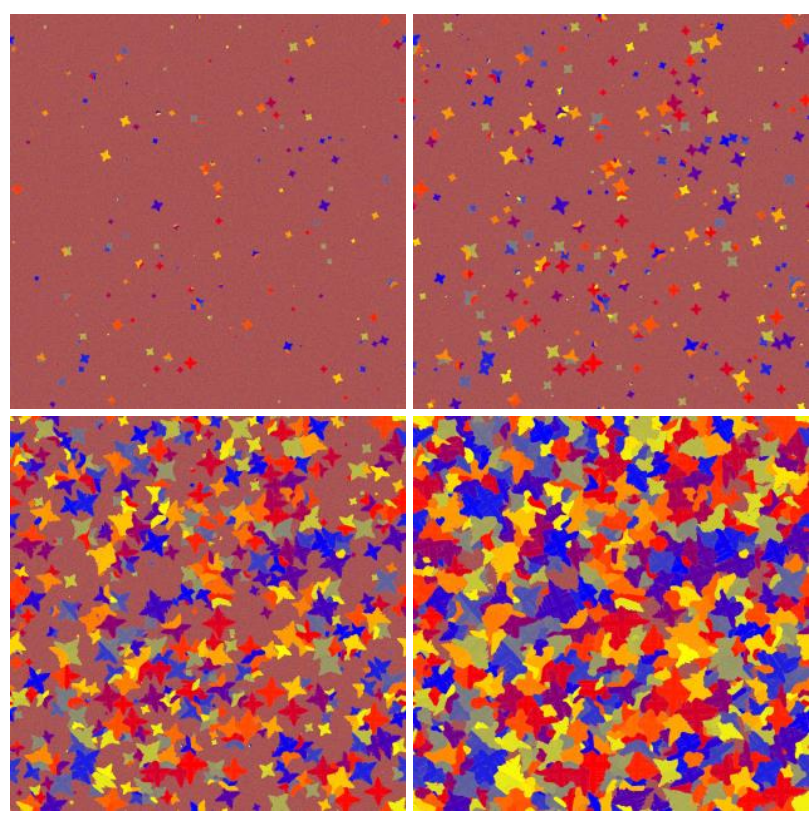

Fig. 5. Nucleation and growth in $2 \mathrm{D}$ within the HMP model. Snapshots of the orientation field taken at $25000,35000,60000$, and 155 000 time steps are shown. The properties of $\mathrm{Cu}-\mathrm{Ni}$ have been used. The EOMs have been solved on a $6144^{2}$ grid.

been assumed, with $s_{0}=0.1$.] The corresponding JohnsonMehl-Avrami-Kolmogorov analysis yields an Avrami-Kolmogorov exponent $m=2.90 \pm 0.04$, a value that falls close to $m=$ 3 corresponding to steady state nucleation combined with 2D steady state growth, found under similar circumstances for the KWC model [17].

Next, we investigated whether the interaction with foreign particles can be handled within the HMP in a similar way as it was done in the case of the KWC model [19]. Here, the foreign particles were handled as 'orientation pinning centers', i.e., randomly positioned pixels, of random but fixed orientation, where the phase field is 0 . A polycrystalline growth form evolved in such system is shown in Fig. 6 that displays the composition-, phase-, and orientations field maps. As in the case of the KWC model, the foreign particles interact with the growing dendrite tips, so that they turn into a modified direction that fits better to the orientation of the foreign particle, after creating a grain boundary, a heterogeneous (particle induced) realization of growth front nucleation. As a result, a disordered (termed 'dizzy') dendrite evolves with arms growing into directions that are not consistent with the orientation of the initial seed.

Work is underway to clarify, whether the homogeneous modes of GFN observed in the case of the KWC model can also be realized within the HMP approach.

\section{Summary}

We have compared the behavior of two phase-field approaches (KWC and HMP described in Refs. [14-16] and [22], respectively) that rely on an orientation field to capture the local crystallographic orientation. We have shown the following:

(i) The two models yield qualitatively similar profiles for the grain boundaries.

(ii) The limiting grain size distributions predicted by the KWC and HMP are similar, and are close to those from the multiphase models of Kim et al. [8] and Schaffnit et al. [9], but deviate perceptibly from the experimental results for thin metallic films [1].

(iii) Adding noise to the equations of motion to represent fluctuations, in HMP simulations we observe nucleation and dendritic growth, with an AvramiKolmogorov exponent of $m=2.90 \pm 0.04$, close to the value corresponding to steady state nucleation accompanied with 2D steady state growth, a result also emerging from the KWC model under similar conditions [17].
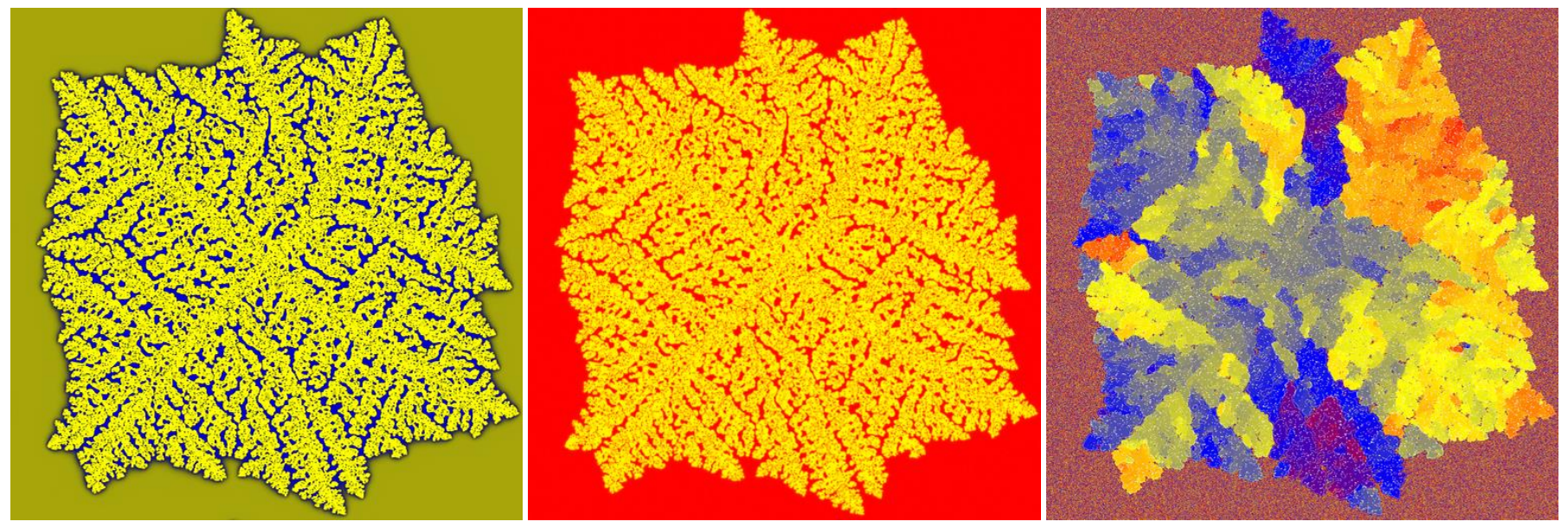

Fig. 6. Interaction of a growing dendrite with foreign particles represented by 'orientation pinning centers' (see text) in the HMP model. From left to right: composition-, phase-field-, and orientation maps are shown. As a result of tip deflection, first described experimentally and theoretically in Ref. [19], the dendrite tips change direction when hitting a foreign particle. In the presence of high particle density this leads to a polycrystalline growth form, in which arms may grow in directions that are not consistent with the orientation of the original seed. The properties of $\mathrm{Cu}-\mathrm{Ni}$ were used. The EOMs have been solved on a $1536^{2}$ grid. 
(iv) We have demonstrated that analogously to the prediction of the KWC model, in the HMP model, the interaction of dendrite tips with foreign particles (represented by orientation pinning centers) lead to the formation of disordered ('dizzy') dendrites.

\section{Acknowledgments}

This work has been supported by the Hungarian-French Bilateral Scientific and Technological Innovation Fund under Grant No. TÉT_12_FR-2-2014-0034; the National Agency for Research, Development, and Innovation (NKFIH), Hungary under contract No. OTKA-K-115959; and by the EU FP7 Collaborative Project "EXOMET" (contract no. NMP-LA-2012280421, co-funded by ESA).

\section{References:}

[1] K. Barmak, E. Eggeling, D. Kinderlehrer, R. Sharp, S. Ta'asan, A.D. Rollett, K.R. Coffey, Prog. Mater. Sci. 58 (2013) 987.

[2] P. Feltham, Acta Metall. 5 (1957) 97.

[3] W.W. Mullins, J. Appl. Phys. 27 (1956) 900.

[4] M. Hillert, Acta Metall. 13 (1965) 227.

[5] L.-Q. Chen and W. Yang, Phys. Rev. B 50 (1994) 15752.

[6] D. Fan, L.-Q. Chen, Acta Mater. 45 (1997) 611.

[7] N. Moelans, B. Blanpain, P.Wollants, Phys. Rev. Lett. 101 (2008) 025502.

[8] S.G. Kim, D.I. Kim, W.T. Kim, Y.B. Park, Phys. Rev. E 74 (2006) 061605.
[9] P. Schaffnit, M. Apel, I. Steinbach, Mater. Sci. Forum 558-559 (2007) 1177.

[10] K.R. Elder, M. Katakowski, M. Haataja, M. Grant, Phys. Rev. Lett. 88 (2002) 245701.

[11] H. Emmerich, H. Löwen, R. Wittkowski, T. Gruhn, G.I. Tóth, G. Tegze, L. Gránásy, Adv. Phys. 61 (2012) 665.

[12] R. Backofen, K. Barmak, K.E. Elder, A. Voigt, Acta Mater. 64 (2014) 72.

[13] L. Gránásy, L. Rátkai, A. Szállás, B. Korbuly, G. I. Tóth, L. Környei, T. Pusztai, Metall. Mater. Trans. A45 (2014) 1694.

[14] R. Kobayashi, J.A. Warren, W.C. Carter, Physica D 119 (1998) 415.

[15] R. Kobayashi, J.A. Warren, W.C. Carter, Physica D 140 (2000) 141.

[16] J.A. Warren, R. Kobayashi, A.E. Lobkovsky, W.C. Carter, Acta Mater. 51 (2003) 6035.

[17] L. Gránásy, T. Börzsönyi, T. Pusztai, Phys. Rev. Lett. 88 (2002) 206105.

[18] L. Gránásy, T. Pusztai, J.A. Warren, J. Phys.: Condens. Matter 16 (2004) R1205.

[19] L. Gránásy, T. Pusztai, J.A. Warren, J.F. Douglas, T. Börzsönyi, V. Ferreiro, Nature Mater. 2 (2003) 92.

[20] L. Gránásy, T. Pusztai, T. Börzsönyi, J.A. Warren, J.F. Douglas, Nature Mater. 3 (2004) 645.

[21] L. Gránásy, T. Pusztai, G. Tegze, J.A. Warren, J.F. Douglas, Phys. Rev. E 72 (2005) 011605.

[22] H. Henry, J. Mellenthin, M. Plapp. Phys. Rev. B 86 (2012) 054117.

[23] J.P Simmons, C. Shen, Y. Wang, Scripta Mater. 43 (2000) 935.

[24] G.I. Tóth, T. Pusztai, L. Gránásy. Phys. Rev. B 92 (2015) 184105 . 